\title{
Use of Fibre Bragg Grating sensors for Gas Electron Multiplier HEP detectors
}

\section{A. Caponero ${ }^{1}$ on behalf of the CMS GEM Collaboration}

ENEA - Centro Ricerche Frascati, Italy and INFN - Laboratori Nazionali di Frascati, Italy

E-mail: michele.caponero@enea.it

The GE1/1 CMS muon system upgrade consists of 144 GEM chambers based on the triple-GEM technology (three GEM foils flat parallel closely spaced). The GE1/1 assembly procedure employs a mechanical stretching procedure to apply tension to the GEM foils by means of a series of lateral stretchers. This novel technology allows mechanical assembly of the GEM chamber without the use of internal spacers or glue. This procedure reduces the assembly time and allows the user to replace one or more GEM foils after the chamber has been assembled. However this mechanical stretching technique needs to be validated to prove that the mechanical stretching technique applies a uniform tensile load on the triple-GEM stack able to fulfil the design specifications which require that foils are planar and uniformly spaced.

The European Physical Society Conference on High Energy Physics 22-29 July 2015

Vienna, Austria

\footnotetext{
${ }^{1}$ Speaker
} 


\section{Introduction}

Fibre Bragg Grating (FBG) sensors already found applications in high energy physics (HEP) for measurement of detector deformation, temperature and humidity. Interest in use of FBGs is due to their specific features, such as small dimensions, long term stability, reduced wiring because of in-series cabling, radiation hardness. In this work we present a novel use of FBG sensors for the mounting procedure of the GE1/1 GEM chambers of the Compact Muon Solenoid (CMS) experiment at the Large Hadron Collider (LHC) of CERN. In the novel application FBG sensors are used to validate the procedure adopted for the installation and tensioning of the wide kapton foils which are inside the active volume of the GEM chamber. According to such procedure the stuck of kapton foils must be installed and stretched plane parallel without the use of glue and internal spacers. With respect to traditional construction techniques, the advantage is both reduced assembly time and the possibility to replace damaged GEM foil after the chamber has been assembled. However the adopted mechanical stretching technique needs to be validated. Preliminary results of the test performed on a full size GE1/1 final prototype and possible future developments are presented.

\section{The Ge1/1 chambers for the CMS upgrade experiment}

The GE1/1 chambers will be installed for the CMS experiment upgrade. In total 144 GEM chambers will be installed in the very forward region of the CMS endcap [1,2]. Each chamber has a trapezium-like surface about $0.5 \times 1.2 \mathrm{~m}^{2}$ wide and has an active volume about $6 \mathrm{~mm}$ high (Figure 1). Chambers are based on the triple GEM technology, which uses a stack of three GEM kapton foils flat stretched parallel to each other. Flatness and parallelism are critical parameters to assure that the performance of the GEM chamber meets the design features with uniform performance across its whole active volume. The design distance between facing kapton foils is $1 \mathrm{~mm}$ and it must be kept uniform with an error of less than $30 \mu \mathrm{m}$. Design spacing between facing foils is obtained by spacers along their contour, with no use of any spacer inside the active volume of the chamber.

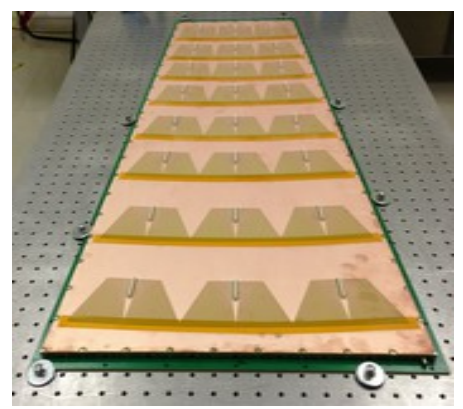

Figure1: GEM chamber being assembled, with readout board in position over the kapton foils.

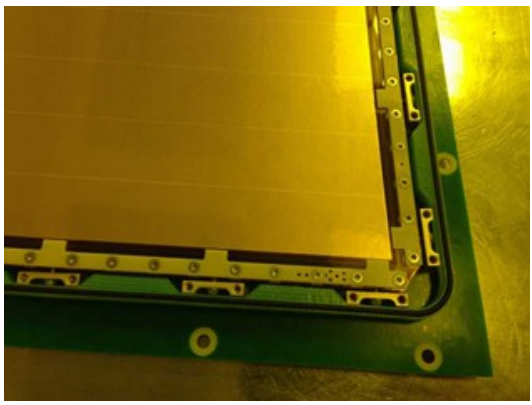

Figure2: Evidence of the external frame and the internal frame (readout board not in position).

To mount a GEM chamber, the three kapton foils must be stretched inside an external trapezium-like aluminium frame which defines the contour of the active volume. The three foils are clamped together along their perimeter by a segmented inner frame which also acts as a spacer and keeps the border of the each foil at the required distance from the border of the 
facing foil (Figure 2). Each segment of the inner frame can be pulled toward the external frame to stretch the three kapton foils all at once. When stretching is adequate and homogeneous the three foils are plane and parallel.

\section{The FBG technology}

FBG sensors are optical sensors embedded in single mode optical fibres; such fibres are about $0.25 \mathrm{~mm}$ in external diameter and can be even many kilometres long. The FBG sensor is about $1 \mathrm{~cm}$ long and is produced by modifying the refractive index of the fibre only where the sensor must be produced. The rest of the fibre is not modified at all and is used to deliver the signal of the FBG sensor. The refractive index modification consist in a periodic modulation of its value along the axis of the fibre. The FBG sensor can be described as a Bragg diffraction grating with effective refraction index $n_{\text {eff }}$ and pitch $\Lambda$. The FBG sensor behaves as a narrowband retro-reflector: when broad-band light propagates along the fibre, a narrow-band signal is generated by diffraction and back-propagates along the fibre. The centre wavelength $\lambda_{\mathrm{B}}$ of the back-propagating narrow-band signal is given by

$$
\lambda_{\mathrm{B}}=2 \Lambda \mathrm{n}_{\mathrm{eff}}
$$

According to equation (1) FBG sensors are sensitive to both temperature and strain. In fact temperature affects $n_{\text {eff }}$ (usually the effect on $\Lambda$ does not need to be considered), whereas strain affects $\Lambda$ (usually the effect on $n_{\text {eff }}$ does not need to be considered). Standard FBGs for sensing applications are written in standard silica telecommunications optical fibre; they are used with broad-band light in the $1500-1600 \mathrm{~nm}$ range and have grating design that produces retro-reflected narrow-band signal about $0.1 \mathrm{~nm}$ FWHM. Such FBGs typical have sensitivity to temperature of about $1 \mathrm{pm} / \mathrm{K}$ and sensitivity to strain of about $1.2 \mathrm{pm} / \mu$ Strain.

Using commercial up-to-date instruments for FBGs interrogation (which provide $1 \mathrm{pm}$ precision), monitoring can be performed with about $0.8 \mu$ Strain precision for strain and $0.1 \mathrm{~K}$ precision for temperature. Many FBGs can be written spaced along one optical fibre, each with a different nominal $\lambda_{B}$ to be operated in wavelength division multiplexing (WDM), with no extra wiring but the single optical fibre on which they are written. Special fibres can be used to write FBGs for special applications in hostile environments, such as high temperature or severe radiation exposure. FBG already found applications in HEP experiments [3, 4].

\section{Scope of the work}

The mounting procedure of the GEM chamber requires that the three kapton foils are stretched plane by acting on screws that pull the segments of the inner frame toward the external frame. Tensioning of the foils is done acting on one segment per time, each segment being controlled by a set of screws. Tensioning of the foils is obtained proceeding all around the frame and starting again, eventually skipping some segment and eventually starting again and again, until uniform stretching is obtained. Tensioning must proceed gradually to avoid that ripples arise because of large stress gradients. No validation of the procedure has been developed so far and the operator can only control the top foil, which is the only one available for visual inspection. The critical issue is to verify the achievement of the planarity of the two not visible 
kapton foils. Use of sensors to monitor in real-time the strain of the kapton foils can validate the procedure.

Sensors must not locally stiffen the tiny foils where they are installed, otherwise false measures would be done. FBG sensors were adopted because they have miniature size and low Young module, so good structural contact with just a little glue and no significant local stiffening can be obtained. Moreover, the FBG technology requires reduced and light wiring, which is important since wires must lay on the tiny foils. Finally, FBG sensors do not require any electrical connection and can have good radiation hardness, which could allow their presence during future tests of the chamber with the active volume being operated.

\section{Experimental method and results}

A full size GE1/1 final prototype was used for the experimental set-up. FBG sensors were installed on the three kapton foils before being mounted in the chamber. Location of sensors is sketched in Figure 3. At each location sensors were installed in pairs, with the two sensors of the pair disposed orthogonal to each other, each of them parallel to one of the two main axis of the foil. That disposition was adopted in order to monitor the strain in the two directions, since FBG sensors are mono-axial. The gage length of each sensor is $10 \mathrm{~mm}$; six sensors are present on each foil; all sensors on the same foil are connected in-series on one optical fiber an plugged to one channel of the electro-optic DAQ device (Figure 4). The position of the sensors identical on the three foils so that the stretching of the three foils can be easily compared.

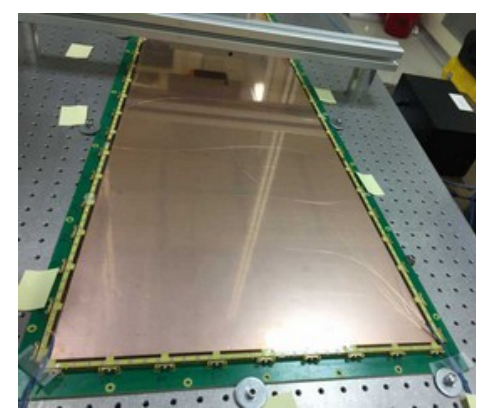

Figure 3: The upper foil with FBGs and optical fibre (barely visible).

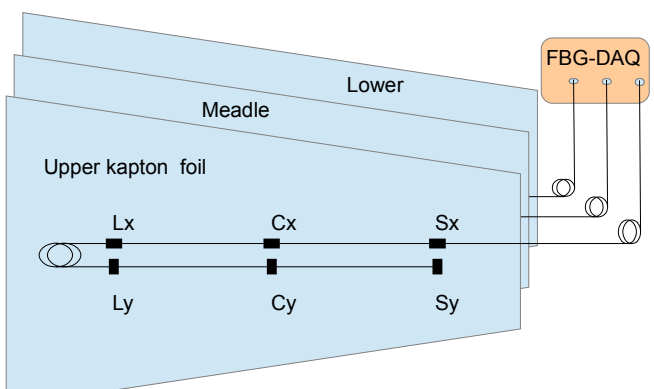

Figure 4: Sketch of the location and nomenclature of the six FBGs installed on each foil.

Before to glue the FBGs on the three kapton foils, gluing tests were performed to select best product and procedure. The issue to be solved was the use of a very thin glue film in order not to locally stiffen the tiny kapton foil and thus false the results. Tested products for gluing include: PWS 24h by UHU; Araldite 2011 by Huntsman, Pattex Plastic by Henkel; UV4028 by Wellomer. Araldite 2011 resulted the best product for the specific application; moreover its radiation hardness properties could also be useful in case of future tests with the GEM chamber being irradiated. After definition of minimal glue thickness for effective structural bonding, a suitable set of tools was developed to assure repetitive glueing procedure with control of glue quantity and effective bonding between foil and FBG.

Foils with the installed FBGs were mounted in the external frame. When foils are loose, local strain is not meaningful because it depends on local bending shape. When foils are straight, local strain is proportional to tensioning force along the axis of the sensor. We briefly recall that strain is the percentage elongation along a line; for materials in elastic axial 
elongation it is proportional to the axial tensioning force via the Young module of the material itself.

Tests were done according to the following procedure in three steps: S1) foils are stretched plane using visual inspection of the upper foil as control of the achieved planarity; S2) tensioning is reduced going to the same condition which applies when foils are mounted in the external frame; S3) foils are stretched back again, adopting visual inspection for complementary control and fine adjustment, but with the leading condition that all sensors of the upper foil tentatively go back to the strain value of step S1.

Result of two tests are given in Figure 5 and Figure 6, which shows the time-history of one triplet of FBGs installed on the three foils with the same in-plane coordinates. The graphs in the two figures are shown as example of the similitude of the results obtained in independent tests, with sensors installed in two different positions and with different orientation. The two graph are fully representative of the results obtained in all tests with any triplet of sensors with the same in-plane coordinates.

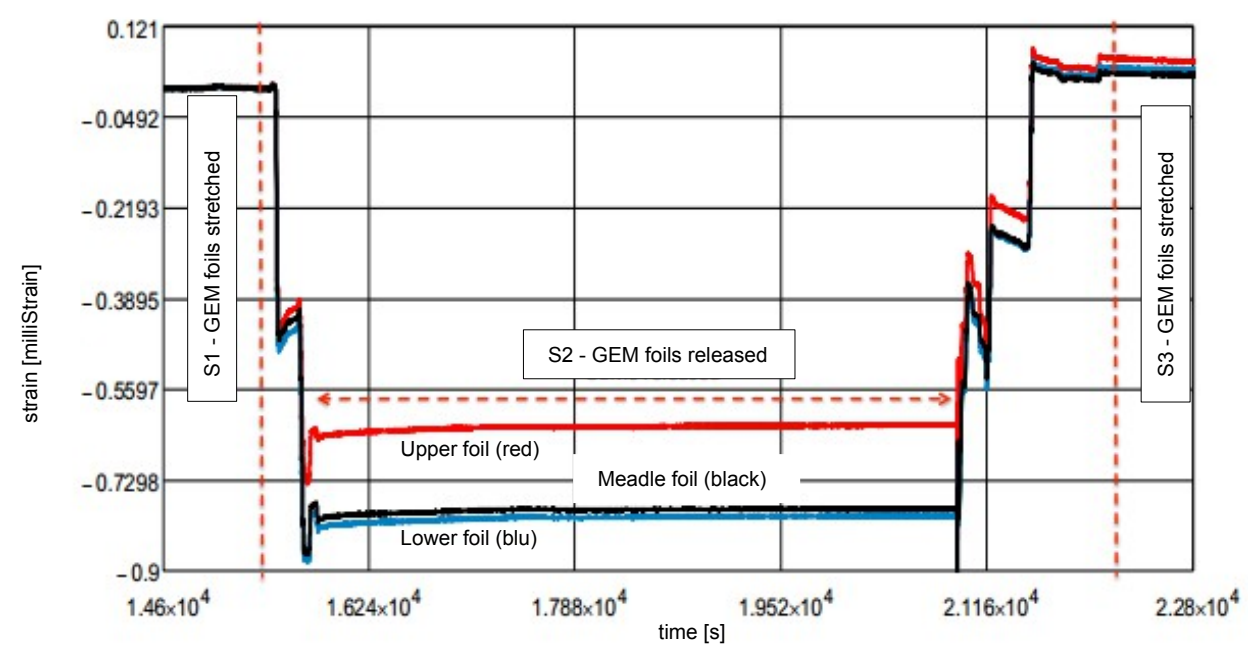

Figure 5: Time History of the three FBGs labelled Cx.

In Figure 5 results are shown for the FBGs labelled Cx. Stretching at step S1 is assumed as 'reference condition' and thus strain measurement is referred to such condition (Strain=0). At step S2 strain goes to lower value showing that foil stretching reduces; different strain values apply to different foils as they fold loose and assume non equal bending conditions. At step S3 value of strain is similar for all foils, showing that they all experience similar stretching, about the value occurring at step S1, thus all foils reach a similar final stretching, despite the previous different loose states.

In Figure 6 results are shown for the FBGs labelled Sy. Similar consideration apply as for Figure 5. The large different variation of the strain from step S1 to step S2 is due to the different position and orientation of the sensors. Graph shows evidence of the evolution of the strain during the step S2. Transitory dips are due to non precise step-by-step tightening of screws during pulling increase, with unwanted partial un-tightening. 


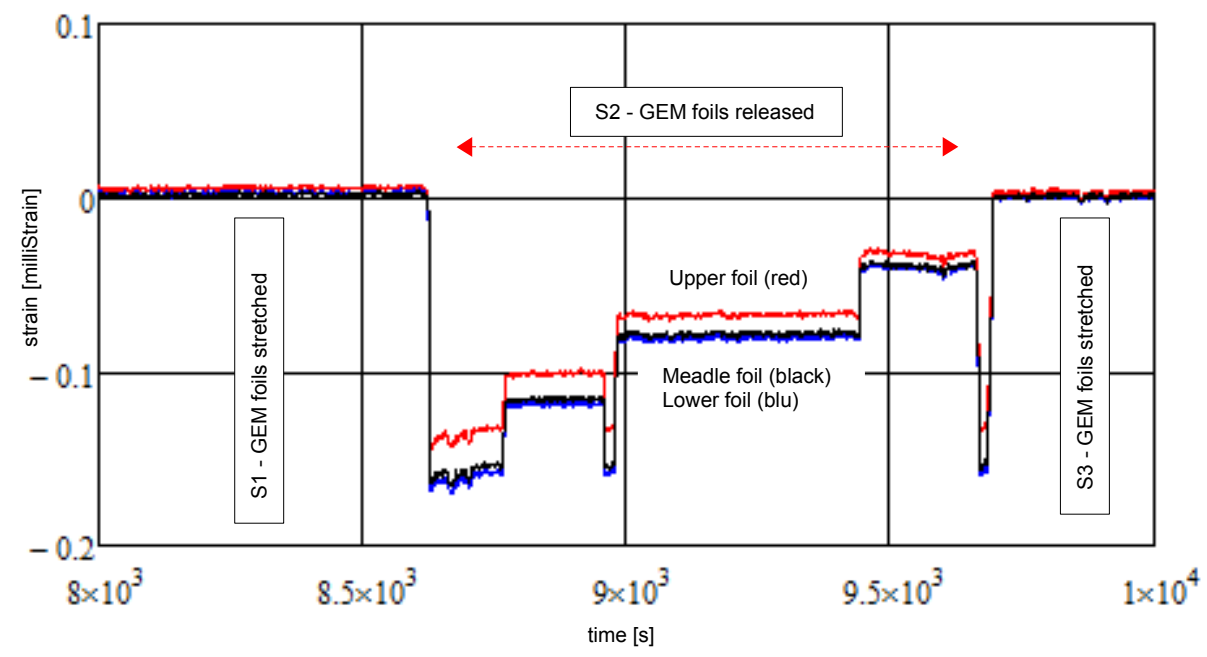

Figure 6: Time History of the three FBGs labelled Sy.

\section{Conclusions and future work}

Use of FBG sensors allowed to validate the mounting and stretching system developed for the three kapton foils of the GE1/1 chambers. The three foils result to be all stretched with similar tensioning despite the previously lay in unevenly loose condition. Use of FBG sensors also allowed to define at what strain foils are straight stretched, which is important not to uselessly overstress the foils with risk of approaching upper safe limit defined for structural integrity.

Future tests are planned to address the definition of a mounting protocol which could guarantee standard and repetitive results with respect to stretching and planarity of foils.

FBG technology features, such as miniature size and light mass, reduced and light wiring, no electrical connection, good radiation hardness, could allow their use for future tests of the chamber with the active volume being operated.

\section{References}

[1] S. Colafranceschi et al., Beam Test Results for New Full-scale GEM Prototypes for a Future Upgrade of the CMS High- $\eta$ Muon. System, in Proc. IEEE NSS-MIC 2012, N14-137, pp. 1172-1176, arXiv:1211.3939v1 [physics.ins-det]

[2] D. Abbaneo et al., Studies on the upgrade of the muon system in the forward region of the CMS experiment at LHC with GEMs, Journal of Instrumentation, 2014, 9, C01053, DOI: 10.1088/1748$0221 / 8 / 12 / \mathrm{C} 12031$

[3] L. Benussi et al., The Omega-like: A novel device using FBG sensors to position vertex detectors with micrometric precision, Nucl Phys. Proc. Suppl. 172 (2007) pp. 263-265, DOI: 10.1016/j.nuclphysbps.2007.08.138

[4] Michele A. Caponero et al., Use of fiber optic technology for relative humidity monitoring in RPC detectors, JINST 8 (2013) T03003, DOI: 10.1088/1748-0221/8/03/T03003 